\title{
Vitamin E reversed nicotine-induced toxic effects on bone biochemical markers in male rats
}

Mohamed Norazlina ${ }^{1}$, Hapidin Hermizi ${ }^{2,3}$, Othman Faizah², Ahmad Shuid Nazrun ${ }^{1}$, Muhammad Norliza ${ }^{1}$, Soelaiman Ima-Nirwana ${ }^{1}$

1Department of Pharmacology, Faculty of Medicine, Universiti Kebangsaan Malaysia, Jalan Raja Muda Abdul Aziz, Kuala Lumpur, Malaysia

2Department of Anatomy, Faculty of Medicine, Universiti Kebangsaan Malaysia, Jalan Raja Muda Abdul Aziz, Kuala Lumpur, Malaysia

${ }^{3}$ Department of Biomedical Science, School of Health Sciences, Universiti Sains Malaysia, Health Campus, Kubang Kerian, Kelantan, Malaysia

Submitted: 20 June 2009

Accepted: 15 October 2009

Arch Med Sci 2010; 6, 4: 505-512

DOI: 10.5114/aoms.2010.14460

Copyright $\odot 2010$ Termedia \& Banach

\section{Abstract}

Introduction: Vitamin E is beneficial in restoring bone histomorphometric parameters in nicotine-treated rats. This study determined the effectiveness of 3 forms of vitamin $\mathrm{E}$ in restoring bone metabolism in nicotine-treated rats.

Material and methods: Thirty-five male Sprague-Dawley rats were divided into 5 groups: (1) control (C), (2) nicotine cessation (NC), (3) $\alpha$-tocopherol (ATF), (4) tocotrienol-enhanced fraction (TEF) and (5) $\gamma$-tocotrienol (GTT). Treatment was carried out for 4 months. The control group was administered normal saline and olive oil throughout the treatment period while treatment for groups 2-5 was performed in 2 phases. In the first phase, the groups received nicotine $7 \mathrm{mg} / \mathrm{kg}$ intraperitoneally for 2 months. The following 2 months, group 2 received normal saline and olive oil while groups 3-5 received ATF, TEF or GTT, $60 \mathrm{mg} / \mathrm{kg}$ orally. Pre-treatment and post-treatment serum was collected for bone biochemical marker measurement using the ELISA method.

Results: Nicotine increased serum bone-resorbing cytokines (interleukin-1 and interleukin-6) and the bone resorption marker pyridinoline (PYD) while reducing the bone formation marker osteocalcin after 2 months of nicotine treatment. The parameters failed to improve after nicotine was stopped for 2 months. Supplementation with the 3 forms of vitamin E improved the parameters, i.e. reduced the cytokines and pyridinoline as well as increased the osteocalcin. In addition, the TEF and GTT groups had a higher level of osteocalcin than the control group.

Conclusions: Nicotine impaired bone metabolism and cessation of nicotine treatment did not reverse the effects. Vitamin E, especially the tocotrienols, restored bone metabolism that was impaired due to nicotine.

Key words: nicotine, vitamin E, bone metabolism, interleukin, rats.

\section{Introduction}

Bone remodelling is a continuous process whereby bone is resorbed by osteoclasts and the new bone is replaced by bone-forming cells, i.e. osteoblasts. [1]. Bone turnover is regulated by many factors such as mechanical forces, hormones (parathyroid and growth hormones), cytokines and local factors [2]. The cytokines, such as interleukins, receptor

\section{Corresponding author:} Ass. Prof. Dr Mohamed Norazlina Department of Pharmacology Faculty of Medicine Universiti Kebangsaan Malaysia Jalan Raja Muda Abdul Aziz 50300 Kuala Lumpur, Malaysia Phone: 603-92897264 Fax: 603-26938205

E-mail: azlina@medic.ukm.my 
activator of nuclear factor $\kappa \mathrm{B}$ ligand (RANKL), tumour necrosis factor- $\alpha$ (TNF- $\alpha)$, interferons and transforming growth factor- $\beta$ (TGF- $\beta$ ), affect bone metabolism by regulating the function of osteoblasts and osteoclasts. For instance, interleukin-1 and interleukin-6 (IL-1 and IL-6) increase osteoclast generation, RANKL inhibits apoptosis of osteoclasts, while TNF- $\alpha$ inhibits osteoblasts [2].

The production of cytokines mentioned above can be influenced by free radicals. A pathological condition such as ovariectomy causes accumulation of reactive oxygen species which in turn promotes the production of TNF- $\alpha$ [3]. In another study, oxidative stress promotes the production of TGF- $\beta$ and osteopontin, which are associated with bone resorption [4]. Thus, bone remodelling is very much dependent on a balance between oxidants and antioxidants. Overwhelming levels of free radicals will disrupt the balance between bone formation and resorption and will lead to bone loss, and hence osteoporosis [5].

Oxidative stress can be induced by many substances, one of which is nicotine [6]. Nicotine has been shown to increase the production of inflammatory mediators [7] and nicotine has been associated with pathogenesis of diseases by promoting proinflammatory mediators [8]. On bone, nicotine induced bone loss and reduced bone mechanical strength in rats [9]. In humans, smoking has been implicated in osteoporosis [10] and is considered one of the risk factors for osteoporosis [11]. Exposure to nicotine has been associated with low bone mineral content in both males and females [12]. In our previous study, we observed that nicotine increased IL-1 and IL-6 [13], and increased pyridinoline but reduced osteocalcin levels significantly [14].

Nicotine-induced osteoporosis may be prevented if the effects of nicotine on promoting oxidative stress can be overcome. This may be made possible by using antioxidants. These antioxidants may act by preventing oxidative stress and curb the overproduction and overactivity of inflammatory mediators such as the interleukins. One of the antioxidants is vitamin E, which exists in 2 forms, i.e. tocopherol and tocotrienol, with 4 isomers each. Vitamin E has been shown to possess antioxidant activities and the activities vary among the different isomers [15]. It suppresses the activation of the inflammatory cascade induced by alcohol [16], reduces the production of IL- 6 by macrophages [17] and may play a role in preventing osteoporosis [18]. Vitamin $\mathrm{E}$ has also been shown to improve the biomarkers of bone metabolism in rats treated with ferric nitrilotriacetate [19]. Palm oil is one of the natural sources of vitamin $E$ and predominantly contains the tocotrienol type of vitamin $\mathrm{E}$. A previous study has shown that palm vitamin $E$, which is mainly composed of tocotrienols, is superior to $\alpha$-tocopherol in protecting bone [20].

Based on the above findings, it is postulated that vitamin E may play a role as a therapeutic agent in preventing bone loss in nicotine-exposed subjects. This study was carried out to determine the effects of vitamin $E$ on the bone of rats exposed to nicotine toxicity. We hypothesized that vitamin E may be able to prevent the adverse effects of nicotine by preventing the overproduction of bone-resorbing cytokines. In this study, a comparison was made between 3 types of vitamin $\mathrm{E}$ preparations, 1) $\alpha$-tocopherol, 2) tocotrienol-enhanced fraction (TEF - a mixture of tocotrienols) and 3) $\gamma$-tocotrienol, to investigate which preparations affect bone the most since different isomers or different preparations of vitamin E might affect a body system differently.

\section{Material and methods}

\section{Animals and treatment}

Thirty-five 3-month old Sprague-Dawley male rats were obtained from the Laboratory Animal Resource Unit, Universiti Kebangsaan Malaysia. The rats were then randomly assigned to 5 groups of 7 rats each and treated for 4 months: (1) control group which received vehicle normal saline and vehicle olive oil (C), (2) nicotine cessation group which received nicotine $7 \mathrm{mg} / \mathrm{kg}$ and vehicle olive oil for the first 2 months followed by vehicle olive oil and vehicle normal saline administration for the following 2 months (NC), (3) $\alpha$-tocopherol group (ATF), (4) tocotrienol-enhanced fraction group (TEF) and (5) $\gamma$-tocotrienol group (GTT). Groups 3-5 received the same nicotine treatment as in group 2 for the first 2 months, but for the following 2 months, the groups received vehicle normal saline and either $\alpha$-tocopherol, tocotrienol-enhanced fraction or $\gamma$-tocotrienol in the dose of $60 \mathrm{mg} / \mathrm{kg}$ respectively.

The rats were housed 3 per cage under $12-\mathrm{h}$ natural light/dark cycles and given tap water ad libitum. All rats received normal rat chow obtained from Gold Coin (Port Klang, Selangor, Malaysia). The nicotine used was nicotine hydrogen tartrate salt which was purchased from Sigma Chemical Co. (St Louis, MO, USA). $\alpha$-Tocopherol acetate was purchased from Sigma Chemical Co. (St Louis, MO, USA) while the Malaysian Palm Oil Board (Bangi, Selangor, Malaysia) supplied the tocotrienolenhanced mixture. The tocotrienol-enhanced mixture was made up of $42.98 \% \alpha$-tocotrienol, $30.98 \% \gamma$-tocotrienol, $13.81 \% \delta$-tocotrienol and $12.23 \%$ palm oil devoid of $\alpha$-tocopherol. $\gamma$-Tocotrienol was a gift from Carotech Berhad (Chemor, Perak, Malaysia). Blood samples were taken before the start of treatment, after 2 months of treatment and at the end of the treatment period. 
Nicotine $7 \mathrm{mg} / \mathrm{kg}$ was prepared by mixing $0.07 \mathrm{~g}$ of nicotine in $10 \mathrm{ml}$ of normal saline. The vitamin $\mathrm{E}$ solutions were prepared by mixing $3 \mathrm{~g}$ of the respective vitamin Es in $50 \mathrm{ml}$ of olive oil (Bertolli, Secaucus, NJ, USA) to get the dose of $60 \mathrm{mg} / \mathrm{kg}$. A total of $0.1 \mathrm{ml} / 100 \mathrm{~g}$ rat weight of the nicotine and vitamin $\mathrm{E}$ preparations were respectively given intraperitoneally and orally via oral gavage, 6 days a week. The nicotine and vitamin $\mathrm{E}$ dosages used in this study have been documented earlier to be effective in causing changes in bone metabolism based on our previous studies [21, 22].

\section{Bone biochemical markers}

Serum IL-1 $\alpha$ and IL- 6 were measured by using rat ELISA kits (Bender MedSystems, Vienna, Austria). Serum osteocalcin was determined by using an ELISA kit purchased from Nordic Bioscience Diagnostic A/S (Herlev, Denmark). Serum pyridinoline was measured by using the METRA EIA kit from Quidel Co. (San Diego, USA).

\section{Serum cotinine levels}

Cotinine, which is a metabolite of nicotine, was measured in the serum using an ELISA kit (DRG Instruments, Germany).

\section{Statistical analysis}

Data analyses were performed by using 'Statistical Package for Social Sciences' (SPSS 12.0). The data were tested for normality using the Kolmogorov-Smirnov test and were found to be normally distributed. The data were analysed by using one-way analysis of variance (ANOVA) followed by Tukey's HSD as the post-hoc test.
Results were presented as mean \pm standard error of the mean (SEM).

The work was conducted in accordance with the Universiti Kebangsaan Malaysia Animal Ethics Committee with the approval number FAR/2003/IMA/12JUNE/095.

\section{Results}

Table I shows the results of all parameters, i.e. serum levels of IL-1, IL-6, osteocalcin and pyridinoline after 2 months' treatment with nicotine. Interleukin- 6 was increased in the NC group at month 2 compared to month 0. IL- 6 was also increased in the TEF group after the $2^{\text {nd }}$ month of nicotine treatment compared to controls. Serum IL-1 was significantly increased after nicotine treatment in all the groups compared to the control group. A similar pattern could be seen in the PYD results. The levels were increased in the treatment groups at month 2 as compared to the control group and also as compared to month 0 within the same groups. Osteocalcin levels were reduced in all treatment groups at month 2 as compared to month 0 . Serum levels of cotinine were also measured and the values increased significantly in NC, ATF, TEF and GTT groups at month 2 as compared to month 0 and differed significantly compared to the control group.

Upon giving TEF or GTT, both IL-1 (Figure 1) and IL-6 (Figure 2) levels were significantly decreased back to normal level. $\alpha$-Tocopherol supplementation was also able to reduce IL-1 levels back to normal value but failed to cause the same effect on IL-6. In addition, IL-1 levels were still high even though nicotine treatment was stopped for 2 months (NC group at month 4).

Table I. Serum levels of bone biomarkers and cotinine in all groups after 2 months of nicotine administration

\begin{tabular}{|ccccccccccc|}
\hline \multirow{2}{*}{ Group } & \multicolumn{2}{c}{ IL-1 [pg/ml] } & \multicolumn{2}{c|}{ IL-6 [pg/ml] } & \multicolumn{2}{c|}{ Osteocalcin [ng/ml] } & Pyridinoline [nmol/l] & \multicolumn{2}{c|}{ Cotinine [ng/ml] } \\
\cline { 2 - 11 } & Month 0 & Month 2 & Month 0 & Month 2 & Month 0 & Month 2 & Month 0 & Month 2 & Month 0 & Month 2 \\
\hline C & 316.57 & 307.41 & 731.30 & 716.34 & 195.22 & 163.34 & 2.47 & 3.71 & 3.0 & 4.0 \\
& \pm 49.16 & \pm 15.36 & \pm 153.84 & \pm 116.68 & \pm 31.59 & \pm 9.94 & \pm 0.24 & \pm 0.45 & \pm 0.3 & \pm 0.3 \\
\hline NC & 198.06 & 566.83 & 262.12 & 456.07 & 287.29 & 119.43 & 2.96 & 5.45 & 2.9 & 56.0 \\
& \pm 18.45 & $\pm 27.67^{* \#}$ & $\pm 13.24^{\#}$ & $\pm 65.62^{*}$ & \pm 8.28 & $\pm 15.96^{*} \#$ & \pm 0.49 & $\pm 0.23^{* \#}$ & \pm 0.5 & $\pm 3.0^{*} \#$ \\
\hline ATF & 267.36 & 598.17 & 513.76 & 704.69 & 214.56 & 120.53 & 2.10 & 6.65 & 3.2 & 54.0 \\
& \pm 58.81 & $\pm 43.92^{* \#}$ & \pm 110.83 & \pm 140.82 & \pm 21.21 & $\pm 9.12^{* \#}$ & \pm 0.13 & $\pm 0.42^{* \#}$ & \pm 0.3 & $\pm 3.0^{* \#}$ \\
\hline TEF & 300.54 & 693.46 & 703.39 & 1052.84 & 237.24 & 118.04 & 2.67 & 6.34 & 2.9 & 54.7 \\
& \pm 56.84 & $\pm 104.08^{* \#}$ & \pm 133.80 & $\pm 111.78^{\#}$ & \pm 31.97 & $\pm 10.25^{* \#}$ & \pm 0.38 & $\pm 0.34^{* \#}$ & \pm 0.5 & $\pm 3.0^{* \#}$ \\
\hline GTT & 304.10 & 624.50 & 502.77 & 901.24 & 237.24 & 118.04 & 2.51 & 6.25 & 3.3 & 55 \\
& \pm 74.00 & $\pm 77.29^{* \#}$ & \pm 91.15 & \pm 114.52 & \pm 31.97 & $\pm 10.25^{* \#}$ & \pm 0.13 & $\pm 0.22^{* \#}$ & \pm 0.5 & $\pm 3.0^{* \#}$ \\
\hline
\end{tabular}

$\mathrm{C}$ - control group supplemented with vehicles i.e. olive oil and normal saline, NC - rats treated with nicotine $7 \mathrm{mg} / \mathrm{kg}$ and olive oil for $2 \mathrm{months}$ and followed by olive oil and normal saline for another 2 months, ATF - rats treated with nicotine $7 \mathrm{mg} / \mathrm{kg}$ and olive oil for 2 months and followed by $\alpha$-tocopherol $60 \mathrm{mg} / \mathrm{kg}$ and normal saline for another 2 months, TEF - rats treated with nicotine $7 \mathrm{mg} / \mathrm{kg}$ and olive oil for 2 months and followed by tocotrienol-enhanced fraction $60 \mathrm{mg} / \mathrm{kg}$ and normal saline for another 2 months, GTT - rats treated with nicotine $7 \mathrm{mg} / \mathrm{kg}$ and olive oil for 2 months and followed by $\gamma$-tocotrienol $60 \mathrm{mg} / \mathrm{kg}$ and normal saline for another 2 months, ${ }^{*}$ indicates significant difference compared to month $O(p<0.05)$, \#indicates significant difference compared to control group of the respective month $(p<0.05)$ 


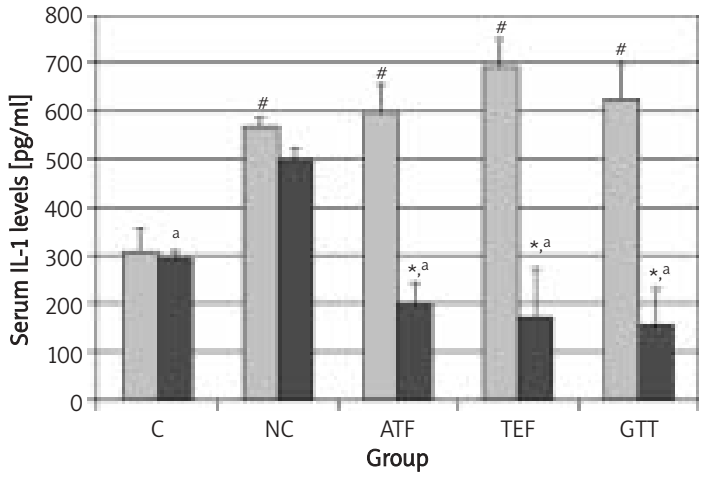

Month 2

Month 4

Figure 1. The effects of vitamin E supplementation on IL-1 levels after 2 months nicotine cessation *indicates significant difference compared to month 2 $(p<0.05)$, \#indicates significant difference compared to control group of the respective month $(p<0.05)$, aindicates significant difference compared to NC group $(p<0.05)$

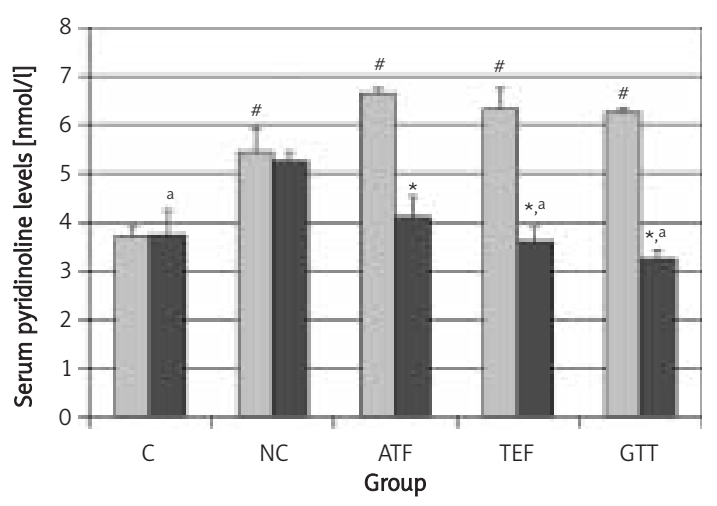

口 Month 2

anth 4

Figure 3. The effects of vitamin E supplementation on pyridinoline levels after 2 months nicotine cessation

*indicates significant difference compared to month 2 $(p<0.05)$, \#indicates significant difference compared to control group of the respective month $(p<0.05)$, aindicates significant difference compared to NC group $(p<0.05)$

The same can be said for PYD levels (Figure 3). The values were persistently high in the NC group even though nicotine treatment was stopped at month 4. On the other hand, at month 4, supplementation of ATF, TEF and GTT significantly reduced PYD levels when compared to month 2 . Furthermore, PYD levels in the TEF and GTT groups were significantly lower than the NC group at month 4. The ATF group did not share this effect.

After 2 months of ATF, TEF and GTT supplementations, i.e. month 4, osteocalcin levels were significantly increased compared to month 2 within the same groups (Figure 4). In addition, osteocalcin

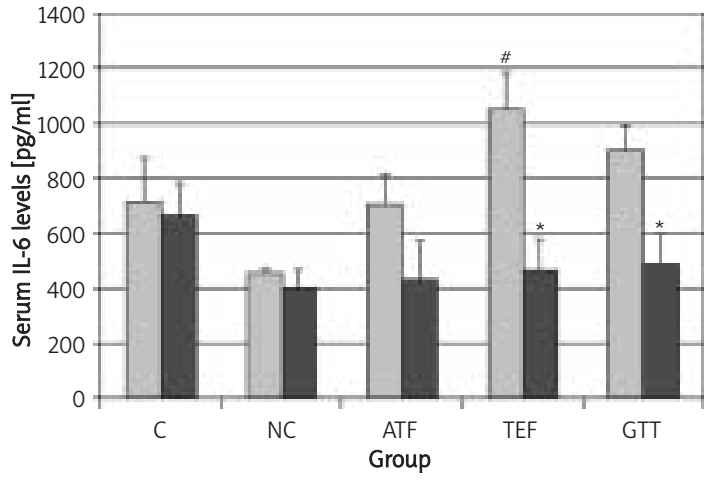

Month 2

Month 4

Figure 2. The effects of vitamin E supplementation on IL- 6 after 2 months nicotine cessation *indicates significant difference compared to month 2 $(p<0.05)$, \#indicates significant difference compared to control group of the respective month $(p<0.05)$, aindicates significant difference compared to NC group $(p<0.05)$

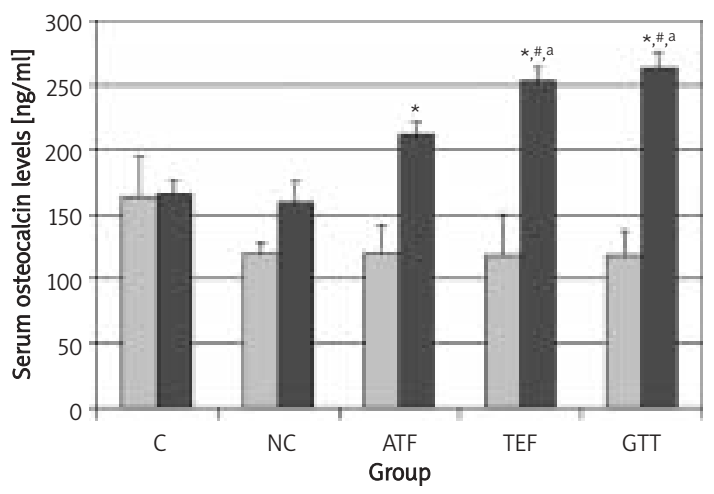

ㅁ Month 2

Month 4

Figure 4. The effects of vitamin E supplementation on osteocalcin levels after 2 months nicotine cessation

*indicates significant difference compared to month 2 $(p<0.05)$, \#indicates significant difference compared to control group of the respective month $(p<0.05)$, aindicates significant difference compared to NC group $(p<0.05)$

levels for TEF and GTT groups at month 4 were significantly higher than the NC group and were even higher than the control group. $\alpha$-Tocopherol did not differ significantly from the NC group or the control group as seen in TEF and GTT groups.

During the second phase of the study, nicotine administration was terminated and cotinine levels reduced in all treatment groups as seen at month 4 (Figure 5).

\section{Discussion}

Nicotine has been shown to induce oxidative stress [23] and oxidative stress has been shown to 
cause bone loss [4]. As a sign of oxidative stress, organs or tissues produce and overexpress inflammatory mediators such as IL-1 and IL-6, as depicted in an earlier study on hepatocytes exposed to ethanol [24].

In the present study, nicotine administration caused an increase in IL-1. Since IL-1 is one of the bone-resorbing cytokines, the increase in this parameter is suggestive of increased bone resorption. Similar findings were observed by Kamer et al. [25], who observed that nicotine induced cytokine release in osteoblast cells. Zhang et al. [26] observed that cigarette smoke increased both IL-1 and IL-6 levels.

Even though in this study we have observed deleterious effects of nicotine on bone parameters, others have found differently. Nicotine administered subcutaneously for 12 weeks did not affect bone mineral density [27], while nicotine given in drinking water for 4 months reduced bone mineral density and affected the mechanical properties of bone [9]. The differences between past studies and ours might be attributed to different dosages and different route of administration.

The rats in this study received nicotine via intraperitoneal injection. In order to ensure that the nicotine injected reached the systemic circulation, we measured the serum levels of cotinine, a metabolite of nicotine. It was found that the cotinine level for the NC group was $56 \mathrm{ng} / \mathrm{ml}$ after 2 months of nicotine administration. An earlier study in humans showed that the serum cotinine level in individuals who smoke 10 to 20 cigarettes per day was $61.59 \mathrm{ng} / \mathrm{ml}$ [28]. We also found low cotinine levels at pre-treatment and at completion of the study in all groups, which might be due to environmental tobacco smoke (ETS).

Interleukin-1 and IL- 6 were found to disturb the enzymatic antioxidant defences, which may lead to oxidative stress [29]. Bone loss due to oxidative stress may be prevented by the administration of antioxidants [30]. In this study, IL-1 was increased due to nicotine treatment and vitamin E supplementation was found to be able to reverse these effects. In comparing between the various vitamin E preparations, all 3 preparations showed similar ability in reversing the nicotine-induced increase in IL-1. This differs from our previous study in which palm tocotrienol mixture was superior to $\alpha$-tocopherol in reversing the increase of IL-1 and IL-6 levels in nicotine-treated [31] and ferricnitrilotriacetate-treated rats [32].

The serum levels of IL- 6 showed inconsistencies. The values were higher in the control group from the beginning of the study. Nicotine treatment (NC group) did not cause an increase in IL-6 levels as compared to the control group. The measurement of IL-6 as an indicator of inflammation is less

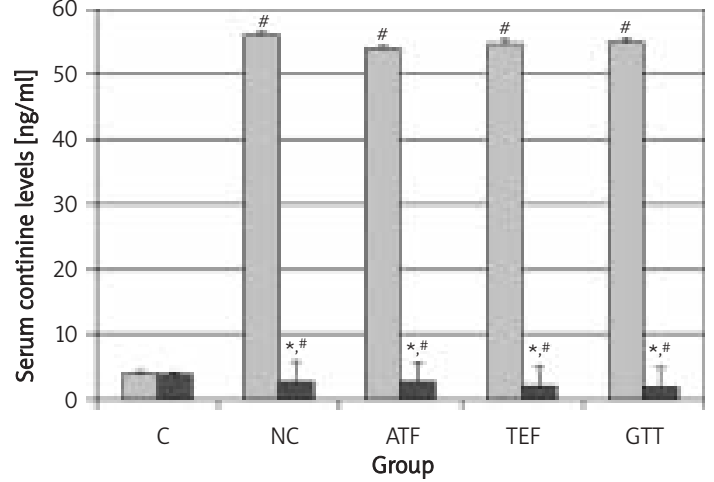

anth 2

Month 4

Figure 5. Serum cotinine levels in rats after 2 months nicotine cessation

*indicates significant difference compared to month 2 $(p<0.05)$, \#indicates significant difference compared to control group of the respective month $(p<0.05)$, aindicates significant difference compared to NC group $(p<0.05)$

reliable than other inflammatory markers [33]. Furthermore, a study on biological variation of IL-6 showed it to have high between-subject coefficient variation, high within-subject coefficient variation, a high individuality index and a low reliability coefficient [34]. This might be the reason for the inconsistent values of IL- 6 seen in this study.

The role of other cytokines must also be considered. Besides interleukins, tumour necrosis factor is another pro-inflammatory cytokine that is involved in stimulating bone resorption [35], and its measurement may be more meaningful. In addition, bone is also maintained by growth factors such as transforming growth factor, which helps in the balance between bone resorption and formation [36]. It was found that whilst IL-1 increases bone resorption, transforming growth factor reduces bone resorption [37]. These factors may contribute to the variations in IL-6 findings in this study.

Vitamin E may exert its effects on bone cytokines via its antioxidant properties. In our previous study, we found that palm tocotrienol mixture was able to reduce lipid peroxidation and increase glutathione peroxidase activity in the femur of normal rats [20]. In other studies, vitamin E has been reported to possess anti-inflammatory activities [38] by suppressing proinflammatory markers [39]. In addition, the immunoregulatory functions of vitamin E during inflammation differ with different vitamin $E$ isomers, as has been shown earlier with tocopherol isomers [40].

The increase of cytokines such as IL- 1 and IL- 6 is associated with osteoclastic bone resorption [41]. In this study, we observed an increase of IL-1 when nicotine was administered and the findings corresponded with the increase in the bone 
resorption marker PYD. Similarly, vitamin E supplementation also reversed the increase in PYD. However, the PYD levels in the ATF group were comparable to the NC group, unlike TEF and GTT, which demonstrated significantly lower PYD values than the NC group. This suggests a lower efficacy of ATF. Our previous study showed that vitamin E was able to reduce levels of the bone-resorbing marker deoxypyridinoline (DPD) in nicotine-induced rats [31]. In rats treated with ferric nitrilotriacetate, palm tocotrienol mixture was better than $\alpha$-tocopherol in reducing the bone resorption marker [32].

The present study also showed that nicotine reduced the levels of osteocalcin (bone formation marker) while vitamin E supplementation was able to revert the levels back to normal. The tocotrienol treated groups (TEF and GTT) had even higher osteocalcin values than the control group. This is suggestive of anabolic actions of vitamin $E$ on bone and requires further study. $\alpha$-Tocopherol did not seem to exert this effect.

Previous studies have shown conflicting results as far as osteocalcin levels are concerned. In one study, administration of $\alpha$-tocopherol acetate to mice increased the synthesis of femoral bone protein as shown by an increase in osteocalcin mRNA [42]. In our previous studies, supplementation of vitamin $\mathrm{E}$ in rats treated with ferric nitrilotriacetate [32] and in rats treated with nicotine [31] did not cause any significant changes in osteocalcin levels. The different outcome observed between this study and our previous studies may be due to the different study design applied. In this study, vitamin E supplementation was given after the oxidant (nicotine) was stopped, while in the previous studies, vitamin E supplementation was given concurrently with the oxidants (ferric nitrilotriacetate and nicotine). This suggests that the antioxidant effect of vitamin $E$ is more evident when the source of oxidant is stopped.

The findings of this study are in accordance with our previous study with regards to bone histomorphometric analysis. Our previous study reported that rats administered nicotine followed by vitamin $E$ supplementation showed an improvement in structural, dynamic and cellular bone histomorphometric parameters as compared to unsupplemented rats [43]. The superiority of tocotrienol-supplemented groups observed in this study was also observed in the previous study, in which supplementation of tocotrienol showed superior effects on bone histomorphometric parameters compared to $\alpha$-tocopherol [43].

$\gamma$-Tocotrienol is shown to be the more potent isomer in reducing serum lipid parameters [44] and in inhibiting adipogenesis [45]. However, in this study, TEF and GTT exert comparable effects on all the parameters measured, indicating that a tocotrienol mixture is just as good as the pure $\gamma$-tocotrienol. In contrast, GTT has been shown previously to exert better effects than TEF on bone histomorphometric parameters in nicotine-induced rats [43].

In this study, the rats were administered nicotine for 2 months and were kept for another 2 months without nicotine treatment. We observed that the pathological processes induced by nicotine on the bone biochemical markers were not reversed when nicotine administration was stopped (as seen in group NC for all parameters). This further confirms that nicotine cessation alone is not sufficient to reverse the adverse effects of nicotine on bone. Vitamin E supplementation may be beneficial in restoring bone homeostasis back to normal.

In conclusion, nicotine treatment for 2 months impaired bone metabolism, which was not reversed following a 2-month nicotine-free period. Vitamin $\mathrm{E}$ supplementation (ATF, TEF and GTT) was able to reverse the effects of nicotine on bone metabolism, most probably due to its immunoregulatory effects. However, different types and isomers of vitamin $E$ may exert different effects on the inflammatory mediators as the tocotrienols were found to be superior to $\alpha$-tocopherol in reversing the effects of nicotine.

\section{Acknowledgments}

The authors are grateful to the Ministry of Science, Technology and Innovation (MOSTI) for funding the research under IRPA grant number 06-02-02-0051-EA243. The authors would like to thank Mr. Faisal Ariffin, Mr. Mohamad Arizi Aziz, Mrs. Azizah Osman, Ms. Mazliadiyana Mazlan and Mr. Mohd. Imran Mohd. Ali for their technical assistance. We also express our gratitude to Mr. Abd Gapor M. Top from the Malaysian Palm Oil Board for supplying the tocotrienol mixture used in this study.

\section{References}

1. Delmas PD, Eastell R, Garnero P, Seibel MJ, Stepan J; Committee of Scientific Advisors of the International Osteoporosis Foundation. The use of biochemical markers of bone turnover in osteoporosis. Osteoporosis Int 2000; 11 (Suppl 6): S2-17.

2. Datta HK, Ng WF, Walker JA, Tuck SP, Varanasi SS. The cell biology of bone metabolism. J Clin Pathol 2008; 61: 577-87.

3. Grassi F, Tell G, Robbie-Ryan M, et al. Oxidative stress causes bone loss in estrogen-deficient mice through enhanced bone marrow dendritic cell activation. Proc Natl Acad Sci USA 2007; 104: 15087-92.

4. Isomura H, Fujie K, Shibata K, et al. Bone metabolism and oxidative stress in postmenopausal rats with iron overload. Toxicology 2004; 197: 93-100.

5. Banfi G, lorio EL, Corsi MM. Oxidative stress, free radicals and bone remodeling. Clin Chem Lab Med 2008; 46: 1550-5. 
6. Chowdury P, Walker A. A cell-based approach to study changes in the pancreas following nicotine exposure in an animal model of injury. Langenbecks Arch Surg 2008; 393: 547-55.

7. Jaimes EA, Tian RX, Joshi MS, Raij L. Nicotine augments glomerular injury in a rat model of acute nephritis. Am J Nephrol 2009; 29: 319-26.

8. Cooper RG, Magwere T. Nitric oxide-mediated pathogenesis during nicotine and alcohol consumption. Indian J Physiol Pharmacol 2008; 52: 11-8.

9. Broulik PD, Rosenkrancova J, Ruzicka P, Sedlacek R, Kurcova I. The effect of chronic nicotine administration on bone mineral content and bone strength in normal and castrated male rats. Horm Metab Res 2007; 39: 20-4.

10. Kapoor D, Jones TH. Smoking and hormones in health and endocrine disorders. Eur J Endocrinol 2005; 152: 491-9.

11. Chapurlat R. Epidemiology of osteoporosis. J Soc Biol 2008; 202: 251-5.

12. Benson BW, Shulman JD. Inclusion of tobacco exposure as a predictive factor for decreased bone mineral content. Nicotine Tob Res 2005; 7: 719-24.

13. Hapidin H, Othman F, Soelaiman IN, Shuid AN, Luke DA, Mohamed N. Negative effects of nicotine on boneresorbing cytokines and bone histomorphometric parameters in male rats. J Bone Miner Metab 2007; 25 : 93-8.

14. Hermizi H, Faizah O, Ima-Nirwana S, Ahmad Nazrun S, Luke DA, Norazlina M. Nicotine impaired bone histomorphometric parameters and bone remodeling biomarkers in Sprague-Dawley male rats. Ann Microsc 2007; 7: 10-24

15. Kamal-Eldin A, Appelqvist LA. The chemistry and antioxidant properties of tocopherols and tocotrienols. Lipids 1996; 31: 671-701.

16. Tiwari V, Kuhad A, Chopra K. Suppression of neuroinflammatory signaling cascade by tocotrienol can prevent chronic alcohol-induced cognitive dysfunction in rats. Behav Brain Res 2009; 203: 296-303.

17. Beharka AA, Han SN, Adolfsson O, et al. Long-term dietary antioxidant supplementation reduces production of selected inflammatory mediators by murine macrophages. Nutr Res 2000; 20: 281-96.

18. Pasco JA, Henry MJ, Wilkinson LK, Nicholson GC, Schneider HG, Kotowicz MA. Antioxidant vitamin supplements and markers of bone turnover in a community sample of nonsmoking women. J Womens Health (Larchmt) 2006; 15: 295-300.

19. Ahmad NS, Khalid BA, Ima-Nirwana S. Effects of vitamin E on interleukin-1 in ferric nitrilotriacetate treated rats. Malaysian J Biochem Biol 2004; 9: 43-7.

20. Maniam S, Mohamed N, Shuid AN, Soelaiman IN. Palm tocotrienol exerted better antioxidant activities than alpha-tocopherol in bone. Basic Clin Pharm Toxicol 2008; 103: 55-60.

21. Norazlina M, Nik-Farideh YMK, Arizi A, Faisal A, ImaNirwana S. Effects of nicotine on bone resorbing cytokines in male rats. Int Med J 2004; 3: www.e-imj.com/Vol3No2/Vol3-No2-B10htm.

22. Norazlina M, Ima-Nirwana S, Gapor MT, Khalid BAK. Palm vitamin $E$ is comparable to alpha-tocopherol in maintaining bone mineral density in ovariectomised female rats. Exp Clin Endocrinol Diab 2000; 108: 305-10.

23. Swami S, Suryakar AN, Katkam RV, Kumbar KM. Absorption of nicotine induces oxidative stress among bidi workers. Indian J Public Health 2006; 50: 231-5.

24. Gutierrez-Ruiz MC, Gomez Quiroz LE, Hernandez E, et al. Cytokine response and oxidative stress produced by ethanol, acetaldehyde and endotoxin treatment in HepG2 cells. Isr Med Assoc J 2001; 3: 131-6.

25. Kamer AR, El-Ghorab N, Marzec N, Margarone JE 3rd, Dziak R. Nicotine induced proliferation and cytokine release in osteoblastic cells. Int J Mol Med 2006; 17: 121-7.

26. Zhang J, Liu Y, Shi J, Larson DF, Watson RR. Side-stream cigarette smoke induces dose-response in systemic inflammatory cytokine production and oxidative stress. Exp Biol Med 2002; 227: 823-9.

27. Akhter MP, Iwaniec UT, Haynatzki GR, Fung YK, Cullen DM, Recker RR. Effects of nicotine on bone mass and strength in aged female rats. J Orthop Res 2003; 21: 14-9.

28. Ziegler UE, Kauczok J, Dietz UA, Reith HB, Schmidt K. Clinical correlation between the consumption of nicotine and cotinine concentrations in urine and serum by competitive enzyme-linked immunosorbent assay. Pharmacol 2004; 72: 254-9.

29. Mathy-Hartert M, Hogge L, Sanchez C, Deby-Dupont G, Crielaard JM, Henrotin Y. Interleukin-1beta and interleukin- 6 disturb the antioxidant enzyme system in bovine chondrocytes: a possible explanation for oxidative stress generation. Osteoarthritis Cartilage 2008; 16: 756-63.

30. Sheweita SA, Khoshhal KI. Calcium metabolism and oxidative stress in bone fractures: role of antioxidants. Curr Drug Metab 2007; 8: 519-25.

31. Norazlina M, Lee PL, Lukman HI, Nazrun AS, Ima-Nirwana S. Effects of vitamin E supplementation on bone metabolism in nicotine-treated rats. Singapore Med J 2007; 48: 195-9.

32. Ahmad NS, Khalid BA, Luke DA, Ima-Nirwana S. Tocotrienol offers better protection than tocopherol from free radicalinduced damage of rat bone. Clin Exp Pharmacol Physiol 2005; 32: 761-70.

33. Tsirpanlis G, Bagos P, Ioannou D, et al. The variability and accurate assessment of microinflammation in haemodialysis patients. Nephrol Dial Transplant 2004; 19 : 150-7.

34. Cava F, González C, Pascual MJ, Navajo JA, GonzálezBuitrago JM. Biological variation of interleukin 6 (IL-6) and soluble interleukin 2 receptor (sIL2R) in serum of healthy individuals. Cytokine 2000; 12: 1423-5.

35. Abdollahi M, Larijani B, Rahimi R, Salari P. Role of oxidative stress in osteoporosis. Therapy 2005; 2: 787-96.

36. Janssens $K$, ten Dijke $P$, Janssens S, Van Hul W. Transforming growth factor beta-1 to the bone. Endoc Rev 2005; 26: 743-74.

37. Kim CH, Kim YH, Kim YK, et al. IL-1beta induces and TGFbeta reduces vitamin $\mathrm{D} 3$-induced bone resorption in mouse calvarial bone cells. Immunol Invest 2003; 32: 171-86.

38. Jiang Q, Ames BN. Gamma-tocopherol, but not alphatocopherol, decreases proinflammatory eicosanoids and inflammation damage in rats. Fed Am Soc Exp Biol J 2003; 17: 816-22.

39. Yam ML, Abdul Hafid SR, Cheng HM, Nesaretnam K. Tocotrienols suppress proinflammatory markers and cyclooxygenase-2 expression in RAW264.7 macrophages. Lipids 2009; 44: 787-97.

40. Berdnikovs S, Abdala-Valencia H, McCary C, et al. Isoforms of vitamin $\mathrm{E}$ have opposing immunoregulatory functions during inflammation by regulating leukocyte recruitment. J Immunol 2009; 182: 4395-405.

41. Mundy GR. Role of cytokines in bone resorption. J Cell Biochem 1993; 53: 296-300.

42. Arjmandi BH, Juma S, Beharka A, Bapna S, Akhter M, Meydani SN. Vitamin E improves bone quality in the aged but not in young adult male mice. J Nutr Biochem 2002; 13: 543-9. 
43. Hermizi H, Faizah O, Ima-Nirwana S, Ahmad Nazrun S, Norazlina M. Beneficial effects of tocotrienol and tocopherol on bone histomorphometric parameters in Sprague-Dawley male rats after nicotine cessation. Calcified Tissue Int 2009; 84: 65-74.

44. Yu SG, Thomas AM, Gapor A, Tan B, Qureshi N, Qureshi AA. Dose-response impact of various tocotrienols on serum lipid parameters in 5-week-old female chickens. Lipids 2006; 41: 453-61.

45. Uto-Kondo H, Ohmori R, Kiyose C, et al. Tocotrienol suppresses adipocyte differentiation and Akt phosphorylation in 3T3-L1 preadipocytes. J Nutr 2009; 139: 51-7. 